\title{
Dietary lipid quality affects temperature-mediated reaction norms of a freshwater key herbivore
}

\author{
Dominik Martin-Creuzburg • Alexander Wacker • \\ Christine Ziese • Martin J. Kainz
}

\begin{abstract}
Temperature-mediated plasticity in life history traits strongly affects the capability of ectotherms to cope with changing environmental temperatures. We hypothesised that temperature-mediated reaction norms of ectotherms are constrained by the availability of essential dietary lipids, i.e. polyunsaturated fatty acids (PUFA) and sterols, as these lipids are involved in the homeoviscous adaptation of biological membranes to changing temperatures. A life history experiment was conducted in which the freshwater herbivore Daphnia magna was raised at four different temperatures $\left(10,15,20,25^{\circ} \mathrm{C}\right)$ with food sources differing in their PUFA and sterol composition. Somatic growth rates increased significantly with increasing temperature, but differences among food sources were obtained only at $10^{\circ} \mathrm{C}$ at which animals grew better on PUFA-rich diets than on PUFA-deficient diets. PUFA-rich food sources resulted in significantly higher population growth rates at $10^{\circ} \mathrm{C}$ than PUFA-deficient food, and the optimum temperature for offspring production was clearly shifted towards colder temperatures with an increased availability of dietary PUFA. Supplementation of PUFA-
\end{abstract}

Communicated by Carla Caceres.

D. Martin-Creuzburg (囚) C. Ziese

Limnological Institute, University of Constance,

Mainaustrasse 252, 78464 Constance, Germany

e-mail: Dominik.Martin-Creuzburg@uni-konstanz.de

A. Wacker

Institute for Biochemistry and Biology, Theoretical Aquatic

Ecology, University of Potsdam, Maulbeerallee 2,

14469 Potsdam, Germany

\section{J. Kainz}

WasserCluster-Biological Station Lunz, Dr. Carl Kupelwieser

Promenade 5, 3293 Lunz am See, Austria deficient food with single PUFA enabled the production of viable offspring and significantly increased population growth rates at $10^{\circ} \mathrm{C}$, indicating that dietary PUFA are crucial for the acclimation to cold temperatures. In contrast, cumulative numbers of viable offspring increased significantly upon cholesterol supplementation at $25^{\circ} \mathrm{C}$ and the optimum temperature for offspring production was shifted towards warmer temperatures, implying that sterol requirements increase with temperature. In conclusion, essential dietary lipids significantly affect temperaturemediated reaction norms of ectotherms and thus temperature-mediated plasticity in life history traits is subject to strong food quality constraints.

Keywords Daphnia $\cdot$ Food quality $\cdot$ Phenotypic plasticity $\cdot$ Polyunsaturated fatty acids $\cdot$ Sterols

\section{Introduction}

Environmental temperature significantly affects phenotypic variation in life history traits of ectothermic animals. In general, ectotherms exposed to lower temperatures reach lower growth rates and mature at a larger body size compared to individuals exposed to warmer temperatures (Atkinson and Sibly 1997; Angilletta et al. 2004). This temperature-mediated plasticity in life history traits, and therewith the ability to grow and survive in an environment with changing temperatures, is subject to physiological constraints. Restructuring the lipid composition of biological membranes in response to changing temperatures is a major strategy by which ectotherms maintain vital physiological functions of membranes, and this homeoviscous adaptation of membrane lipids potentially affects the capability of ectotherms to cope with varying temperatures 
(Sinensky 1974; Hazel and Prosser 1974). Acclimatisation to low environmental temperatures in ectothermic animals is often associated with an increase in the proportion of mono- or polyunsaturated fatty acids (PUFA) within membrane phospholipids and, concomitantly, with a decrease in the proportion of saturated fatty acids (Hazel and Williams 1990). The degree of unsaturation of membrane phospholipids, i.e. the number and position of double bonds within phospholipid acyl chains, can have pronounced effects on membrane properties (Hazel and Williams 1990). Long chain PUFA, i.e. PUFA with 20 or more carbon atoms, show a tendency to accumulate in cold-exposed and winter-active ectotherms, suggesting that they play an important role in the acclimation/adaptation to low temperatures (Farkas 1979; Schlechtriem et al. 2006; Smyntek et al. 2008).

In general, animals are incapable of synthesising long chain PUFA de novo and hence a potential acclimation to cold temperatures may depend on the availablity of dietary PUFA. A deficiency in dietary PUFA has been shown to constrain growth and reproduction of ectotherms (von Elert 2002; Becker and Boersma 2003; Ravet et al. 2003; MartinCreuzburg et al. 2009). In general, five PUFA are considered to be essential: the three omega- $3(n-3)$ fatty acids $\alpha$-linolenic acid (ALA, 18:3n-3), eicosapentaenoic acid (EPA, 20:5n-3), and docosahexaenoic acid (DHA, 22:6n-3) and the two omega-6 (n-6) fatty acids linoleic acid (LIN, 18:2n-6) and arachidonic acid (ARA, 20:4n-6). EPA, DHA, and ARA have also been termed as 'conditionally dispensable' (Cunnane 2000) as some animals are capable of converting these PUFA from their respective precursors ALA and LIN. However, such conversion rates are often insufficient to meet physiological demands and thus dietary sources of these PUFA are required (Von Elert 2002; Leonard et al. 2004).

Cholesterol is another indispensable structural component of cell membranes and as such is involved in the regulation of membrane fluidity, permeability, and the functioning of various membrane proteins (Haines 2001; Ohvo-Rekilä et al. 2002). Altering the cholesterol content of cell membranes provides another mechanism by which organisms can adjust to changing environmental temperatures (Hazel and Williams 1990; Crockett 1998). In particular at warmer temperatures, the static order of biological membranes is stabilised by cholesterol and the cholesterol content of membranes and animal tissues is often positively correlated with growth temperature (Hazel and Williams 1990; Hassett and Crockett 2009; Sperfeld and Wacker 2009). In contrast to plants and algae, which contain a large variety of different sterols (phytosterols), cholesterol is the predominant sterol in animal tissues. In herbivorous animals, it is synthesised from dietary phytosterols, though not all phytosterols are suitable precursors for cholesterol (Behmer and Nes 2003; Martin-Creuzburg and von Elert 2009). Consequently, in ectotherms that are unable to synthesise cholesterol de novo (e.g. all arthropods), one would expect that the requirements for suitable dietary sterols increase with increasing temperature (Hassett and Crockett 2009; Sperfeld and Wacker 2009).

Using the freshwater herbivore Daphnia magna as model organism, we investigated food quality effects on life history traits at different temperatures to assess how temperature-mediated reaction norms of ectothermic animals are affected by the availability of essential dietary lipids. For this purpose, a life history experiment was conducted at four different temperatures at which juvenile animals were raised on algae (Scenedesmus obliquus, Nannochloropsis limnetica, Cryptomonas sp.) that differed in their PUFA and sterol composition. In addition, the lipid composition of the moderate food source $S$. obliquus, which is poor in PUFA and sterols that are conducive for somatic growth and reproduction, was experimentally modified by PUFA and sterol supplementation. We hypothesised that dietary PUFA supply gains in importance for somatic growth and reproduction with decreasing temperature, while dietary sterol supply gains in importance with increasing temperatures.

\section{Materials and methods}

\section{Cultivation of algae}

The green alga Scenedesmus obliquus (SAG 276-3a) was used as food for the stock cultures of Daphnia magna, it was grown in batch cultures in Cyano medium (Jüttner et al. 1983). S. obliquus, the eustigmatophyte Nannochloropsis limnetica (SAG 18.99), and the cryptophyte Cryptomonas sp. (SAG 26.80) were used as food for the Daphnia growth experiment. Algae were cultivated semi-continuously in aerated 5 -L vessels at $20^{\circ} \mathrm{C}$, illumination at $150 \mu \mathrm{mol}$ quanta $\mathrm{m}^{-2} \mathrm{~s}^{-1}$, and a dilution rate of $0.25 \mathrm{D}^{-1}$ either in Cyano medium (S. obliquus, N. limnetica) or in Woods Hole (WC) medium with vitamins (Cryptomonas sp.). Although there were no indications that the food quality for Daphnia was affected by the addition of vitamins to the algal growth medium, we cannot completely rule out that the observed differences in food quality among the three algae were to some extent biased by the availability of vitamins (see, e.g., Keating 1985). Food suspensions of these algae were obtained by centrifugation and resuspension in fresh medium. Carbon concentrations of the food suspensions were estimated from photometric light extinctions $(800 \mathrm{~nm})$ and from previously established regressions between carbon and light extinction determined in dilution series of each food suspension. The light extinction regressions were confirmed by subsequent carbon analysis of the food suspensions. 
Liposome preparation

Liposome stock suspensions were prepared from $3 \mathrm{mg}$ 1-palmitoyl-2-oleoyl-phosphatidylglycerol (POPG) and $7 \mathrm{mg}$ 1-palmitoyl-2-oleoyl-phosphatidylcholin (POPC; Lipoid, Germany) dissolved in an aliquot of ethanol. PUFA- or cholesterol-containing liposomes were prepared by adding $3.33 \mathrm{mg}$ of PUFA (ARA, EPA, or DHA) or cholesterol (all Sigma) from lipid stock solutions in ethanol. The resulting solutions were further processed as described in Martin-Creuzburg et al. (2009).

\section{Growth experiments}

Stock cultures of a clone of $D$. magna were raised in filtered lake water $(0.2-\mu \mathrm{m}$ pore-sized membrane filter) with saturating concentrations $\left(2 \mathrm{mg} \mathrm{C} \mathrm{L}{ }^{-1}\right)$ of $S$. obliquus. The growth experiment was conducted in climate chambers (16:8 $\mathrm{h}$ light:dark cycle) using third-clutch juveniles born within $12 \mathrm{~h}$ in glass beakers filled with $200 \mathrm{~mL}$ of filtered lake water. Animals were exposed to constant temperatures of $10,15,20$, and $25^{\circ} \mathrm{C}$, respectively, to cover a temperature range Daphnia species experience in the field. Algae were supplied at non-limiting concentrations $(3 \mathrm{mg}$ $\left.\mathrm{C} \mathrm{L}^{-1}\right)$. Lipid supplementation was achieved by adding $60 \mu \mathrm{L}$ of a PUFA- or cholesterol-containing liposome stock suspension to a corresponding beaker. To test for possible effects of the supplemented liposomes, $60 \mu \mathrm{L}$ of a liposome stock suspension prepared without adding PUFA or sterols were used in a control treatment (cf. MartinCreuzburg et al. 2009). Each treatment consisted of three replicates with seven juvenile $D$. magna per beaker. Every day, daphnids were transferred into new beakers with freshly prepared food suspensions. On day 5 , three daphnids were subsampled from each beaker, dried for $24 \mathrm{~h}$, and weighed (Mettler Toledo XP2U; $\pm 0.1 \mu \mathrm{g}$ ). The juvenile somatic growth rates $(g)$ were determined as the increase in dry weight from the beginning of the experiment $\left(W_{0}\right)$ to day $5\left(W_{t} ; t=\right.$ age in days):

$g=\frac{\ln W_{t}-\ln W_{0}}{t}$.

The remaining daphnids were kept in corresponding treatments until they had released their third-clutch juveniles. The number of viable offspring was determined in each successive reproduction cycle. Population growth rates $(r)$ were estimated iteratively using the Euler-Lotka equation:

$1=\sum_{x=0}^{n} l_{x} m_{x} \mathrm{e}^{-r x}$,

where $l_{x}$ is the age-specific survivorship, $m_{x}$ is the agespecific fecundity (total number of neonates divided by adult individuals in each beaker), and $x$ is the age at reproduction (in days). The probability of survival until reproduction $\left(l_{x}\right)$ was estimated from the mortality that occurred in the different treatments. Growth rates were calculated as means of each treatment. In addition to population growth rates, the cumulative numbers of viable offspring produced within the first three reproduction cycles were evaluated to assess the reproductive success of animals exposed to different temperatures and dietary quality.

\section{Analyses}

For the analysis of dietary fatty acids and sterols, $\sim 1 \mathrm{mg}$ particulate organic carbon (POC) was filtered separately onto precombusted GF/F filters (Whatman, $25 \mathrm{~mm}$ ). Filters were deposited in $7 \mathrm{~mL}$ of dichloromethane:methanol (2:1, $\mathrm{v:v}$ ) and stored at $-20^{\circ} \mathrm{C}$. Total lipids were extracted, using three washes, from sonicated filters with dichloromethane:methanol $(2: 1, v: v)$. Pooled cell-free extracts were evaporated to dryness under $\mathrm{N}_{2}$-atmosphere. The lipid extracts were transesterified with methanolic $\mathrm{HCl}$ ( $3 \mathrm{~mol} \mathrm{~L}{ }^{-1}, 60^{\circ} \mathrm{C}, 15 \mathrm{~min}$ ) for the analysis of fatty acids or saponified with methanolic $\mathrm{KOH}\left(0.2 \mathrm{~mol} \mathrm{~L}^{-1}, 70^{\circ} \mathrm{C}, 1 \mathrm{~h}\right)$ for the analysis of sterols. Subsequently, fatty acid methyl esters (FAME) were extracted $3 \times$ with iso-hexane $(2 \mathrm{~mL})$; the neutral lipids were partitioned into iso-hexane:diethyl ether $(9: 1, v: v)$. The lipid-containing fraction was evaporated to dryness under $\mathrm{N}_{2}$ and resuspended in iso-hexane (10-20 $\mu \mathrm{L})$. Lipids were analysed by gas chromatography (GC; Hewlett-Packard $6890^{\mathrm{TM}}$ ) equipped with a flame ionization detector (FID) and a DB-225 [J\&W Scientific, $30 \mathrm{~m} \times 0.25 \mathrm{~mm}$ inner diameter (i.d.) $\times 0.25 \mu \mathrm{m}$ film] capillary column for FAME analysis and with a HP-5 (Agilent, $30 \mathrm{~m} \times 0.25 \mathrm{~mm}$ i.d. $\times 0.25 \mu \mathrm{m}$ film) capillary column for sterol analysis. Details of GC configurations are given elsewhere (Martin-Creuzburg et al. 2009, 2010). Lipids were quantified by comparison to internal standards (C17:0 ME and C23:0 ME; $5 \alpha$-cholestan) of known concentrations, considering response factors determined previously with lipid standards (Sigma or Steraloids). Lipids were identified by their retention times and their mass spectra, which were recorded with a GC-mass spectrometer (GCQ, Finnigan MAT) equipped with a fused-silica capillary column (DB-225MS, J\&W for FAMEs; DB-5MS, Agilent for sterols; GC configurations as described for FID). Sterols were analysed in their free form and as their trimethylsilyl derivatives, which were prepared by incubating $20 \mu \mathrm{L}$ of iso-hexane sterol extract with $10 \mu \mathrm{L}$ of $\mathrm{N}, \mathrm{O}$-bis(trimethylsilyl)trifluoroacetamide (BSTFA) including $1 \%$ trimethylchlorosilane (TMCS) for $1 \mathrm{~h}$ at room temperature. Spectra were recorded between 50 and $600 \mathrm{amu}$ in the electron impact (EI) ionization mode. The 
limit for quantitation of fatty acids and sterols was $20 \mathrm{ng}$. The absolute amount of each lipid was related to the particulate organic carbon (POC), which was determined as described in Martin-Creuzburg et al. (2009).

\section{Data analysis}

Data were analysed using the statistical software package $\mathrm{R}$ (v.2.11). Somatic and population growth rates of $D$. magna obtained with different food sources were plotted against the reciprocal temperature values (which are commonly used to analyse temperature dependent rates; Dixit and Ray 1982) and the slopes of the obtained linear regression models were compared using a multiple comparison $q$ test according to Zar (2010) and classified into homogenous groups $(P<0.05)$. Intercepts of the different regression models were not evaluated statistically as they did not represent ecologically relevant temperatures. In addition to linear regression models, differences in somatic and population growth rates between food treatments were analysed separately at the lowest and the highest temperature (i.e. at 10 and $25^{\circ} \mathrm{C}$ ) using one-way analyses of variance (ANOVA). Raw data met the assumption of homogeneity of variance; treatment effects were tested by Tukey's honestly significant difference (HSD) tests.

The nonlinear regressions between temperature and cumulative numbers of viable offspring were analysed using the following optimum function:

$y=a\left(T-x_{\max }\right)^{2}+y_{\max }$,

where $y$ is the cumulative number of viable offspring, $a$ the slope of the parabola, $T$ the temperature, $x_{\max }$ the optimum temperature for offspring production, and $y_{\max }$ the maximum number of viable offspring produced at $x_{\max }$ within the first three reproduction cycles. Data were fitted to the equation using the function nls of R v.2.11. Differences in $x_{\text {max }}$ and $y_{\max }$ between food treatments were then assessed by comparing the obtained $95 \%$ confidence intervals. Oneway ANOVA was also used to analyse differences in the cumulative numbers of viable offspring produced by D. magna at 10 and $25^{\circ} \mathrm{C}$ in the different food treatments.

\section{Results}

Biochemical composition of the food sources

The fatty acid composition of $S$. obliquus was characterised by high amounts of LIN and ALA and by the absence of PUFA $>20$ carbon atoms (Table 1 ). In contrast, $N$. limnetica contained considerable amounts of LIN and ARA, and exceptionally high amounts of EPA (Table 1). The fatty acid composition of Cryptomonas $\mathrm{sp}$. was characterised by high amounts of LIN, ALA, stearidonic acid (SDA; 18:4n3 ), and EPA, moderate amounts of DHA, and by the absence of ARA (Table 1). Three sterols could be detected in $S$. obliquus (IUPAC names in parentheses): fungisterol ( $5 \alpha$-ergost-7-en- $3 \beta$-ol), chondrillasterol $[(22 E)$ - $5 \alpha$-poriferasta-7,22-dien-3 $\beta$-ol], and 22-dihydrochondrillasterol ( $5 \alpha$ -

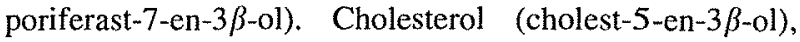
24 -ethylcholesterol $(24 \alpha$ or $\beta$ ), and (iso)fucosterol [ $24 Z$ or $E$-stigmasta-5,24(28)-dien-3 $\beta$-ol] were the principal sterols found in $N$. limnetica (Table 1). The C-24 stereochemistry of 24-ethylcholesterol, i.e., either sitosterol $(24 \alpha$; stigmast5 -en-3 $\beta$-ol) or clionasterol $(24 \beta$; poriferast-5-en-3 $\beta$-ol), and the cis trans isomers fucosterol $(E)$ and isofucosterol $(Z)$ could not be identified with certainty. Stigmasterol (22E-stigmasta-5,22-dien-3 $\beta$-ol) and (epi)brassicasterol ( $24 \alpha ; 22 E$-campesta-5,22-dien-3 $\beta$-ol or $24 \beta ; 22 E$-ergosta5,22 -dien-3 $\beta$-ol) were the principal sterols found in Cryptomonas $\mathrm{sp}$.

Liposomes packed with single PUFA or cholesterol contained considerable amounts of these PUFA or cholesterol, but neither cholesterol nor any PUFA were found in liposomes prepared without supplementing these compounds (Table 1).

\section{Temperature-dependent growth responses of D. magna}

Juvenile somatic growth rates of $D$. magna increased considerably with increasing temperature, but the slopes of the linear regression models did not differ among the three different food algae or among supplemented treatments (Fig. 1; Table 2). In addition to the linear regression analyses, differences in somatic growth rates between food treatments were analysed at the lowest and the highest temperature, i.e. at 10 and $25^{\circ} \mathrm{C}$. At $10^{\circ} \mathrm{C}$, somatic growth rates of D. magna fed Cryptomonas sp. were significantly higher than those of animals fed $S$. obliquus (Tukey's HSD, $P<0.05$ following ANOVA, $F_{7,16}=5.70, P=0.002$; Fig. 1), but somatic growth rates obtained with $N$. limnetica did not differ significantly from those obtained with $S$. obliquus or Cryptomonas sp. (Tukey's HSD, $P>0.05$ ). At $25^{\circ} \mathrm{C}$, somatic growth rates did not differ significantly between food treatments (ANOVA, $F_{7,16}=2.42$, $P=0.068$; Fig. 1). The supplementation of $S$. obliquus with single PUFA or cholesterol did not significantly improve somatic growth rates of $D$. magna at any temperature (Tukey's HSD, $P>0.05$; Fig. 1).

As with somatic growth rates, population growth rates of $D$. magna increased considerably with increasing temperatures. In contrast, however, the slopes of the obtained linear regression models differed significantly between food sources (Fig. 2; Table 2). The linear regressions between temperature and population growth rates of $D$. magna fed $N$. limnetica or Cryptomonas sp. were 
Table 1 Polyunsaturated fatty acid (PUFA) and sterol content of $S$. obliquus, N. limnetica and Cryptomonas sp.; data represent means of three

replicates \pm standard deviation

Food suspensions consisting of S. obliquus and PUFA- or cholesterol-containing liposomes contained either $26.7 \pm 2.7$ ARA, $27.3 \pm 3.7$ EPA, $30.4 \pm 1.3$ DHA, or $19.0 \pm 4.7$ cholesterol, respectively (all values in $\mu \mathrm{g} \mathrm{mg} \mathrm{C}{ }^{-1} \pm \mathrm{SD}$ ) n.d. Not detectable

\begin{tabular}{llll}
\hline & $\begin{array}{l}\text { S. obliquus } \\
\left(\mu \mathrm{g} \mathrm{mg} \mathrm{C}^{-1}\right)\end{array}$ & $\begin{array}{l}\text { N. limnetica } \\
\left(\mu \mathrm{g} \mathrm{mg} \mathrm{C}^{-1}\right)\end{array}$ & $\begin{array}{l}\text { Cryptomonas sp. } \\
\left(\mu \mathrm{g} \mathrm{mg} \mathrm{C}^{-1}\right)\end{array}$ \\
\hline $18: 2 \mathrm{n}-6$ (LIN) & $22.2 \pm 2.7$ & $13.3 \pm 1.2$ & $21.0 \pm 1.9$ \\
$18: 3 \mathrm{n}-6$ & $2.3 \pm 1.9$ & $0.8 \pm 0.3$ & n.d. \\
$18: 3 \mathrm{n}-3$ (ALA) & $95.4 \pm 14.0$ & $0.9 \pm 0.5$ & $92.0 \pm 8.4$ \\
$18: 4 \mathrm{n}-3$ & $7.0 \pm 0.5$ & n.d. & $49.0 \pm 4.7$ \\
$20: 2 \mathrm{n}-6$ & n.d. & $1.1 \pm 0.9$ & n.d. \\
20:3n-6 & n.d. & $0.6 \pm 0.1$ & n.d. \\
20:4n-6 (ARA) & n.d. & $23.2 \pm 1.5$ & n.d. \\
20:5n-3 (EPA) & n.d. & $192.8 \pm 18.3$ & $43.4 \pm 6.7$ \\
22:6n-3 (DHA) & n.d. & n.d. & $8.3 \pm 0.8$ \\
(n-3) PUFA & $102.5 \pm 14.4$ & $194.1 \pm 16.8$ & $184.4 \pm 19.7$ \\
(n-6) PUFA & $24.5 \pm 4.5$ & $39.0 \pm 4.1$ & $21.0 \pm 1.9$ \\
(n-3):(n-6) & $4.2 \pm 0.2$ & $5.0 \pm 0.7$ & $8.8 \pm 0.2$ \\
Total PUFA & $127.0 \pm 18.8$ & $233.1 \pm 16.7$ & $205.4 \pm 21.6$ \\
Cholesterol & n.d. & $7.3 \pm 0.4$ & n.d. \\
Sito- or clionasterol & n.d. & $2.0 \pm 0.6$ & n.d. \\
(iso)Fucosterol & n.d. & $0.9 \pm 0.4$ & n.d. \\
(epi)Brassicasterol & n.d. & n.d. & 5.3 \\
Stigmasterol & n.d. & n.d. & n.7 \pm 0.5 \\
Fungisterol & $1.4 \pm 0.2$ & n.d. & n.d. \\
Chondrilasterol & $4.2 \pm 0.4$ & n.d. & n.d. \\
22-Dihydrochondrillasterol & $0.7 \pm 0.1$ & n.d. & n.d. \\
Total sterols & $6.3 \pm 0.4$ & $10.2 \pm 0.8$ & $13.0 \pm 1.7$ \\
\hline & & &
\end{tabular}

characterised by significantly lower slopes than the regression obtained with dietary $S$. obliquus (Table 2), which is mostly due to the significantly higher population growth rates obtained with $N$. limnetica or Cryptomonas sp. at $10^{\circ} \mathrm{C}$ (Tukey's HSD, $P<0.05$ following ANOVA, $F_{7,16}=44.35, P<0.001$; Fig. 2). Likewise, supplementation of $S$. obliquus with ARA, EPA or DHA enabled the production of viable offspring and resulted in significantly increased population growth rates at $10^{\circ} \mathrm{C}$ (Tukey's HSD, $P<0.05)$ and, consequently, in lower slopes of the linear regressions between temperature and population growth rates (Fig. 2; Table 2). Effectively, at $10^{\circ} \mathrm{C}$, population growth rates increased upon ARA or EPA supplementation up to the level obtained with $N$. limnetica as food. In contrast, supplementation of $S$. obliquus with cholesterolcontaining liposomes did not affect population growth rates of D. magna at $10^{\circ} \mathrm{C}$ (Tukey's HSD, $P>0.05$; Fig. 2) and the slopes of the regression models did not differ (Table 2). At $25^{\circ} \mathrm{C}$, population growth rates obtained with $N$. limnetica were significantly lower than those obtained with S. obliquus, whereas population growth rates obtained with Cryptomonas sp. were significantly higher than those obtained with $S$. obliquus (Tukey's HSD, $P<0.05$ following ANOVA, $F_{7,16}=66.99, P<0.001$; Fig. 2). At $25^{\circ} \mathrm{C}$, supplementation of $S$. obliquus with control liposomes or DHA-containing liposomes did not affect population growth rates of D. magna (Tukey's HSD, $P>0.05$; Fig. 2). However, population growth rates on a $S$. obliquus diet slightly but significantly increased upon ARA and EPA supplementation at $25^{\circ} \mathrm{C}$ (Tukey's HSD, $P<0.05$; Fig. 2). The supplementation of $S$. obliquus with cholesterol did not significantly increase population growth rates at $25^{\circ} \mathrm{C}$ (Tukey's HSD, $P=0.086$; Fig. 2).

The cumulative numbers of viable offspring produced by $D$. magna were significantly affected by temperature and food treatments. Temperature-dependent offspring production was analysed using non-linear regression models (Fig. 3), and differences between the optimum functions obtained with the different food sources were then assessed by comparing the $95 \%$ confidence intervals of the calculated optimum temperature for offspring production $\left(x_{\max }\right)$ and the maximum numbers of viable offspring $\left(y_{\max }\right.$; Table 3$)$. In general, at any temperature, the cumulative numbers of viable offspring produced by D. magna were highest when fed Cryptomonas sp. (Fig. 3). The regression model revealed that the highest numbers of viable offspring were produced by animals fed Cryptomonas sp. at a temperature of $16.5^{\circ} \mathrm{C}$ (Fig. 3; Table 3). In contrast, with $N$. limnetica as food, the highest numbers of viable offspring were produced at $11.8^{\circ} \mathrm{C}$ and with a 
Fig. 1 Juvenile somatic growth rates of $D$. magna

(means $\pm \mathrm{SD}, n=3$ ) raised at four temperatures $(10,15,20$,

$25^{\circ} \mathrm{C}$ ) on different food sources. D. magna were fed

unsupplemented a Scenedesmus obliquus (solid line and circles), Cryptomonas sp. (long dashed line and triangles) or Nannochloropsis limnetica (short dashed line and squares); or $S$. obliquus supplemented with $b$ control liposomes or liposomes containing $\mathbf{c}$ ARA, d EPA, e DHA or $f$ cholesterol (supplemented diets represented by dashed-dotted lines and open circles). For comparison, somatic growth rates obtained with unsupplemented $S$. obliquus are plotted in each panel. Somatic growth rates were plotted against the reciprocal temperature values and the slopes of the obtained linear regression models were analysed statistically. For somatic growth rates, slopes did not differ significantly between food sources (N.S. not significant)
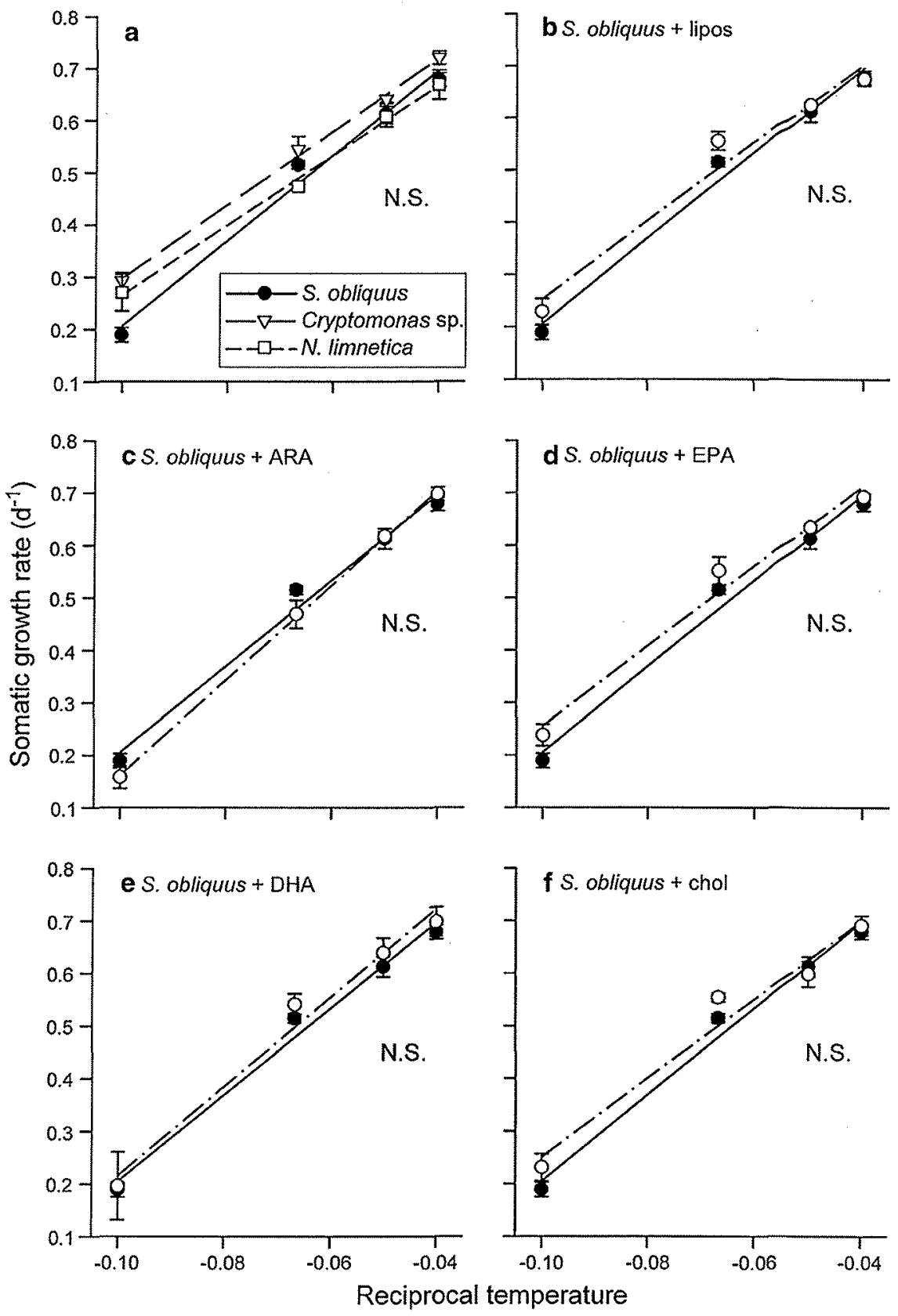

S. obliquus as food at $18.8^{\circ} \mathrm{C}$ (Fig. 3; Table 3), whereas the maximum numbers of viable offspring produced with $N$. limnetica or $S$. obliquus did not differ. Hence, the optimum temperature for offspring production differed significantly between food sources (confidence intervals of the calculated temperatures did not overlap). Supplementation of $S$. obliquus with ARA or EPA increased the production of viable offspring at any temperature (Fig. 3), and the maximum numbers of viable offspring $\left(y_{\max }\right)$ did not differ significantly between the Cryptomonas sp. diet and the ARA- or EPA-supplemented diets (Table 3).
However, the positive effect of ARA and EPA supplementation was most evident at $10^{\circ} \mathrm{C}$ because at this temperature animals were unable to produce viable offspring without ARA or EPA supplementation (Fig. 3). At $10^{\circ} \mathrm{C}$, $D$. magna fed unsupplemented $S$. obliquus or $S$. obliquus supplemented with control liposomes or cholesterol-containing liposomes produced eggs, but these eggs were stripped off with exuviae during molting, remained at the bottom of the beakers and did not further develop (only a single viable offspring was observed, when unsupplemented or cholesterol-supplemented $S$. obliquus was used 
Table 2 Comparison of linear regression models used to analyse temperature-dependent somatic and population growth rates of $D$. magna fed different food sources

\begin{tabular}{|c|c|c|c|c|c|c|}
\hline \multirow[t]{2}{*}{ Treatment } & \multicolumn{3}{|c|}{ Somatic growth rate $(\mathrm{g})$} & \multicolumn{3}{|c|}{ Population growth rate $(r)$} \\
\hline & $R^{2}$ & Slopes (CI) & Groups & $R^{2}$ & Slopes (CI) & Groups \\
\hline S. obliquus & 0.98 & $8.20(7.43-8.96)$ & $A, B$ & 0.99 & $6.92(6.32-7.51)$ & A \\
\hline N. limnetica & 0.98 & $6.71(6.03-7.39)$ & $\mathrm{B}$ & 0.99 & $3.66(3.37-3.96)$ & $\mathrm{C}$ \\
\hline Cryptomonas sp. & 0.99 & $7.06(6.58-7.54)$ & B & 0.97 & $4.94(4.32-5.56)$ & $\mathrm{B}$ \\
\hline S. obliquus + lipos & 0.96 & $7.51(6.43-8.59)$ & $\mathrm{A}, \mathrm{B}$ & 1.00 & $6.59(6.36-6.82)$ & A \\
\hline S. obliquus + ARA & 0.99 & $9.05(8.55-9.55)$ & A & 0.98 & $5.30(4.70-5.89)$ & $\mathrm{B}$ \\
\hline S. obliquus + EPA & 0.97 & $7.63(6.75-8.51)$ & $A, B$ & 0.98 & $5.36(4.89-5.84)$ & $\mathrm{B}$ \\
\hline S. obliquus + DHA & 0.95 & $8.45(7.16-9.75)$ & $A, B$ & 0.98 & $5.46(4.86-6.06)$ & $\mathrm{B}$ \\
\hline S. obliquus + cholesterol & 0.96 & $7.47(6.35-8.58)$ & $A, B$ & 0.99 & $7.19(6.80-7.59)$ & A \\
\hline
\end{tabular}

Slopes of the different regression models were compared using a $q$ test and classified into homogenous groups, i.e. different letters indicate significantly different groups $(P<0.05$, all lql values $>5.0) .95 \%$ confidence intervals of slope estimations are provided in parentheses (all $t$ values $>14.56$; all $P$ values $<0.001$ ). Intercepts of the different regression models were not evaluated statistically as they did not represent ecologically relevant temperatures. Animals fed $S$. obliquus supplemented with control liposomes did not produce any viable offspring at $10^{\circ} \mathrm{C}$ and hence population growth rates could not be calculated using Eq. (2). Thus, population growth rates of animals fed $S$. obliquus supplemented with control liposomes at $10^{\circ} \mathrm{C}$ were calculated using the offspring numbers produced by animals fed unsupplemented $S$. obliquus at $10^{\circ} \mathrm{C}$ in order to obtain the full temperature range for all food treatments for statistical evaluations. This is a conservative assumption because no reproduction would end up in even more negative population growth rates and would increase the significance of the differences to the fatty acid supplementation treatments

as food). At any temperature, offspring production slightly increased upon DHA supplementation, but the maximum numbers of viable offspring $\left(y_{\max }\right)$ did not differ significantly between $S$. obliquus and the DHA supplemented diet (Fig. 3; Table 3). The optimum temperature for offspring production $\left(x_{\max }\right)$ was not significantly affected by PUFA supplementation (Fig. 3; Table 3). In contrast, the supplementation of $S$. obliquus with cholesterol significantly increased offspring production at $25^{\circ} \mathrm{C}$ (Tukey's HSD, $P<0.05$ following ANOVA, $F_{7,16}=51.92, P<0.001$; Fig. 3), and, as a consequence, the optimum temperature for offspring production $\left(x_{\max }\right)$ was shifted towards warmer temperatures (from 18.8 to $20.2^{\circ} \mathrm{C}$; Table 3 ).

The age at reproduction (defined as the days needed to release offspring) was significantly affected by temperature but not by food treatments. With increasing temperature, the age at first reproduction decreased on average from 24 , 12,9 , to 7 days at $10,15,20$, and $25^{\circ} \mathrm{C}$, respectively. Maternal mortality was marginal (only five animals died during the experiment) and not affected by food or temperature.

\section{Discussion}

Temperature-mediated reaction norms for somatic and population growth of $D$. magna were differently affected by the quality of the available food. A comparison of juvenile somatic growth rates revealed significant differences only at the lowest test temperature. It was suggested that dietary PUFA play a substantial role for somatic growth in particular at colder temperatures (Masclaux et al. 2009) and that EPA growth saturation thresholds are higher at low compared to high temperatures (Sperfeld and Wacker 2011), which is in accordance with our finding that the PUFA-rich algae Cryptomonas sp. and $N$. limnetica allowed for higher somatic growth rates at $10^{\circ} \mathrm{C}$ (statistically significant only for Cryptomonas sp.). However, this hypothesis was challenged by the finding that supplementation of S. obliquus with single PUFA did not significantly increase somatic growth rates of D. magna at $10^{\circ} \mathrm{C}$, which suggests that somatic growth was constrained by other factors than dietary lipids at this temperature.

The availability of dietary PUFA gains in importance in later life stages when animals increase their investment in reproduction (Martin-Creuzburg et al. 2009, 2010). As with juvenile somatic growth rates, population growth rates of D. magna increased considerably with increasing temperature. In contrast, however, the temperature-mediated reaction norms of population growth rates were significantly affected by the quality of the available food. In particular at $10^{\circ} \mathrm{C}$, population growth rates of animals fed Cryptomonas sp. or $N$. limnetica were significantly higher than those of animals fed $S$. obliquus, suggesting that dietary long chain PUFA are crucial for biomass production at colder temperatures. This implication is corroborated by the finding that supplementation of $S$. obliquus with single PUFA (ARA, EPA, or DHA) significantly increased population growth rates at $10^{\circ} \mathrm{C}$ and, consequently, by a lower slope of the applied linear regression model. At $10^{\circ} \mathrm{C}$, population growth rates of animals fed $S$. obliquus increased upon ARA or EPA supplementation up 
Fig. 2 Population growth rates of $D$. magna (means $\pm \mathrm{SD}$, $n=3$ ) raised at four temperatures $\left(10,15,20,25^{\circ} \mathrm{C}\right)$ on different food sources. D. magna were fed unsupplemented a Scenedesmus obliquus (solid line and circles), Cryptomonas sp. (long dashed line and triangles) or Nannochloropsis limnetica (short dashed line and squares); or $S$. obliquus supplemented with b control liposomes or liposomes containing $\mathrm{c}$ ARA, d EPA, e DHA or $f$ cholesterol (supplemented diets represented by dashed-dotted lines and open circles). For comparison, population growth rates obtained with unsupplemented S. obliquus are plotted in each panel. Population growth rates were plotted against the reciprocal temperature values and the slopes of the obtained linear regression models were analysed statistically (for details, see Table 2); significant $P$ values are shown, N.S. not significant. Animals fed S. obliquus supplemented with control liposomes (b) did not produce any viable offspring at $10^{\circ} \mathrm{C}$ and hence population growth rates could not be calculated (for details, see Table 2)
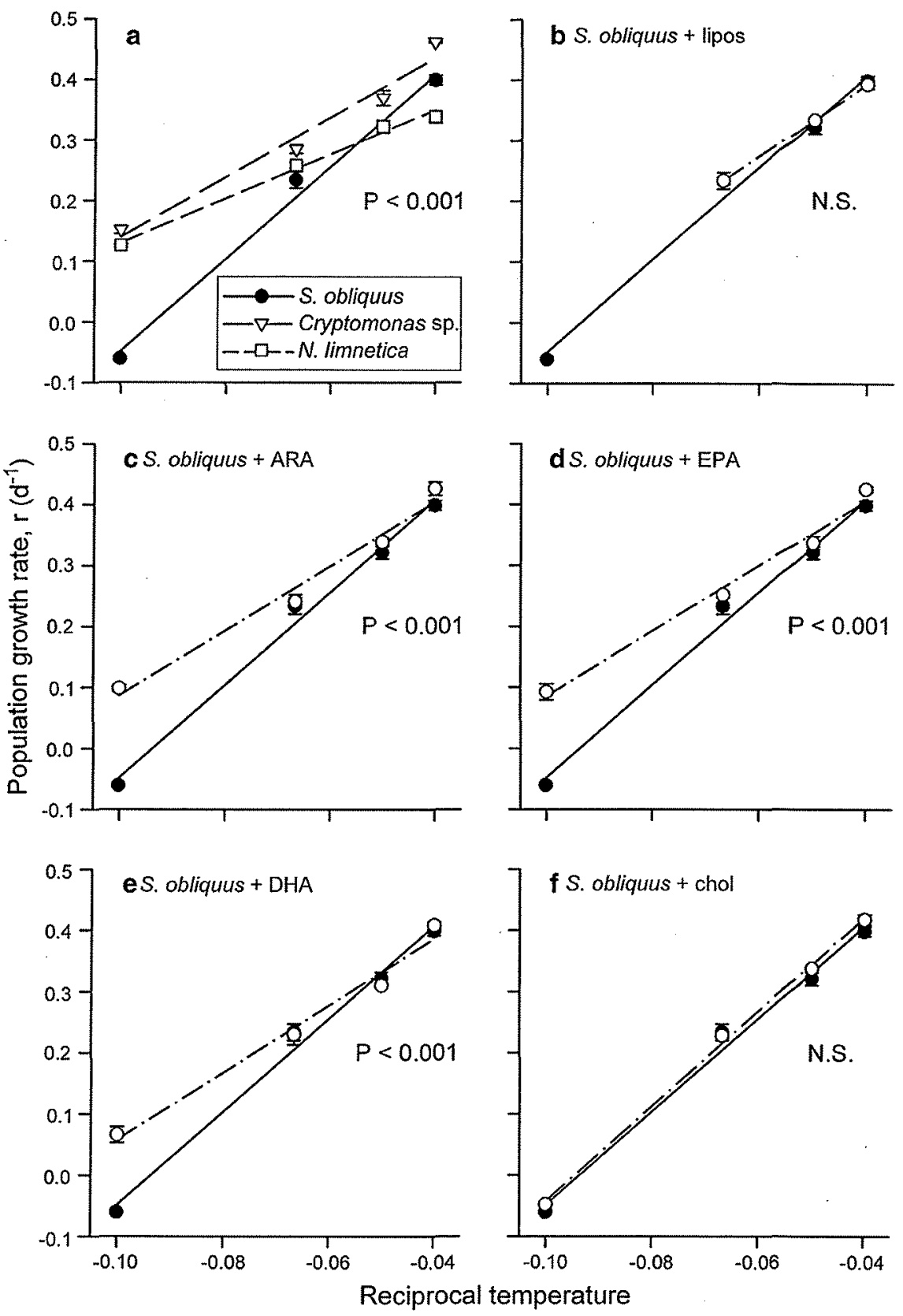

to the level obtained with $N$. limnetica and almost up to the level obtained with Cryptomonas sp., which indicates that differences in food quality among the three algae are at this temperature primarily due to differences in their PUFA composition.

At $25^{\circ} \mathrm{C}$, however, population growth rates of animals fed $S$. obliquus were higher than those of animals fed $N$. limnetica, resulting in an intersection of the temperaturemediated reaction norms. Hence, the dietary quality of the two algae for $D$. magna changed differently with temperature. At $25^{\circ} \mathrm{C}$, population growth rates on a $S$. obliquus diet increased slightly upon ARA and EPA supplementation, indicating that these long chain PUFA also support population growth at higher temperatures. Besides their significance for membrane physiology, ARA and EPA serve as precursors for eicosanoids (e.g. prostaglandins), locally acting hormones, which in both vertebrates and invertebrates are important mediators in reproduction, the immune system, and ion transport physiology (Stanley 2000; Heckmann et al. 2008). Considering that not all the multiple functions ARA and EPA assume in animal physiology are related to temperature acclimation, this 
Fig. 3 Cumulative numbers of viable offspring produced by D. magna (means $\pm \mathrm{SD}, n=3$ ) in the first three reproduction cycles. Animals were raised at four temperatures $(10,15,20$, $25^{\circ} \mathrm{C}$ ) on different food sources. D. magna were fed unsupplemented a Scenedesmus obliquus (solid line and circles), Cryptomonas sp. (long dashed line and triangles) or

Nannochloropsis limnetica (short dashed line and squares); or $S$. obliquus supplemented with b control liposomes or liposomes containing $\mathrm{c}$ ARA, d EPA, e DHA or $\mathbf{f}$ cholesterol (supplemented diets represented by dashed-dotted lines and open circles). For comparison, cumulative numbers of viable offspring produced with unsupplemented $S$. obliquus are plotted in each panel. Offspring numbers and the temperature optima for offspring production were analysed using a nonlinear regression model (see Table 3)
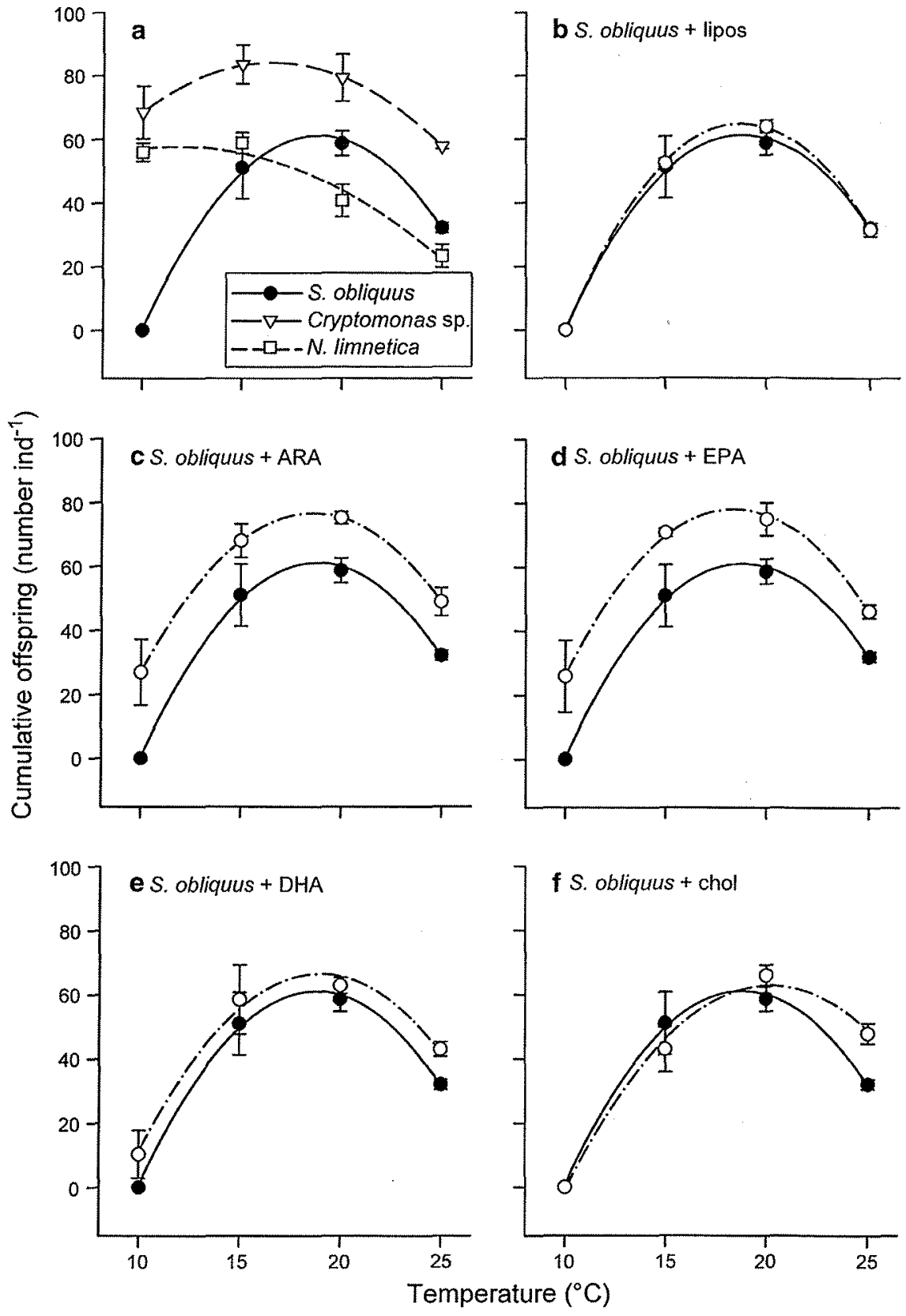

implies that a dietary source of these PUFA is also required at warmer temperatures. Recently, it has been shown that both dietary ARA and EPA strongly improve population growth rates of $D$. magna on a PUFA-free diet at $20^{\circ} \mathrm{C}$ (Martin-Creuzburg et al. 2010). Supplementation of $S$. obliquus with DHA also significantly increased population growth rates of $D$. magna at $10^{\circ} \mathrm{C}$, albeit this positive effect was less pronounced than the effect obtained upon ARA or EPA supplementation. The tendency of DHA to accumulate in winter-active poikilotherms suggests that this PUFA plays a crucial role in the adaptation to low temperatures (Hazel and Williams 1990; Smyntek et al. 2008). However, as dietary DHA is effectively converted to EPA in Daphnia (von Elert 2002; Martin-Creuzburg et al. 2010), the positive effect of DHA supplementation on population growth rates is presumably the result of an increased availability of EPA.

The cumulative numbers of viable offspring produced by $D$. magna were significantly affected by both temperature and food treatments. At any temperature, the cumulative numbers of viable offspring produced by $D$. magna were highest with Cryptomonas sp. as food, indicating that 
Table 3 Parameters of the nonlinear regression models (Eq. 3) used to analyse the effects of temperature on the cumulative numbers of viable offspring produced by $D$. magna within the first three reproduction cycles with different food sources: $a$ is the slope of the parabolic optimum function (all $t$ values $<-3.86$; except for
$N$. limnetica $(P=0.004)$, all $P$ values $<0.001), x_{\max }$ is the optimum temperature for offspring production (all $t$ values $>7.41$; all $P$ values $<0.001$ ), and $y_{\max }$ is the maximum number of viable offspring produced per individual (all $t$ values $>21.61$; all $P$ values $<0.001$ )

\begin{tabular}{|c|c|c|c|c|c|c|}
\hline Treatment & $R^{2}$ & $a$ & $x_{\max }\left({ }^{\circ} \mathrm{C}\right)$ & Groups $x_{\max }$ & $y_{\max }\left(\right.$ no. ind $\left.{ }^{-1}\right)$ & Groups $y_{\max }$ \\
\hline S. obliquus & 0.96 & $-0.77(-0.91$ to -0.64$)$ & $18.84(18.39-19.29)$ & A & $61.08(55.84-66.32)$ & A \\
\hline N. limnetica & 0.92 & $-0.20(-0.32$ to -0.08$)$ & $11.79(8.19-15.39)$ & B & $57.66(53.40-61.93)$ & A \\
\hline Cryptomonas sp. & 0.78 & $-0.37(-0.52$ to -0.21$)$ & $16.50(15.45-17.54)$ & $\mathrm{C}$ & $84.11(77.93-90.29)$ & $\mathrm{B}$ \\
\hline S. obliquus + lipos & 0.99 & $-0.84(-0.89$ to -0.80$)$ & $18.76(18.63-18.88)$ & A & $64.66(63.05-66.27)$ & A \\
\hline S. obliquas + ARA & 0.93 & $-0.67(-0.83$ to -0.52$)$ & $18.59(18.02-19.15)$ & A & $76.58(70.51-82.65)$ & B \\
\hline S. obliquus + EPA & 0.93 & $-0.74(-0.89$ to -0.58$)$ & $18.39(17.88-18.90)$ & A & $78.10(70.90-84.30)$ & $\mathrm{B}$ \\
\hline S. obliquus + DHA & 0.92 & $-0.68(-0.86$ to -0.50$)$ & $19.01(18.30-19.72)$ & $A, D$ & $66.54(59.57-73.50)$ & $A, B$ \\
\hline S. obliquus + cholesterol & 0.97 & $-0.61(-0.73$ to -0.48$)$ & $20.23(19.51-20.96)$ & $\mathrm{D}$ & $62.82(58.22-67.41)$ & A \\
\hline
\end{tabular}

95\% confidence intervals are provided in parentheses. Confidence intervals of $x_{\max }$ and $y_{\max }$ were used to group food treatments, i.e. different letters indicate that confidence intervals did not overlap. $R^{2}$ indicates the proportion of variance explained

Cryptomonas sp. is of superior food quality for D. magna. The regression model revealed that the optimum temperatures for offspring production differed significantly among the three algae tested. Interestingly, this optimum shifted towards colder temperatures with an increased availability of dietary PUFA, as total PUFA concentrations of the three food algae increased from $S$. obliquus, Cryptomonas sp., to $N$. limnetica, again suggesting that dietary PUFA play a vital role in temperature acclimation, in particular for the ability to grow and reproduce at colder temperatures. The temperature range at which high numbers of cumulative offspring were produced was smallest on a $S$. obliquus diet and, compared to Cryptomonas sp. and N. limnetica, clearly shifted towards warmer temperatures. $S$. obliquus contained high amounts of C18 PUFA, but no PUFA with $>18$ carbon atoms. With $N$. limnetica as food, the temperature range at which high numbers of cumulative offspring were produced was clearly shifted towards colder temperatures. In fact, the food quality of $N$. limnetica, as judged by offspring production, decreased with increasing temperature. As $N$. limnetica contains exceptionally high EPA concentrations, decreasing offspring production with increasing temperature might be due to toxic products generated by the temperature-mediated peroxidation of excess dietary EPA (Crockett 2008; see also Watson et al. 2009 for toxic effects of dietary EPA).

The supplementation of $S$. obliquus with ARA or EPA significantly increased the production of viable offspring at any temperature, and the maximum numbers of viable offspring increased upon ARA or EPA supplementation up to the level obtained with Cryptomonas sp. as food. Although the optimum temperature for offspring production was not affected by PUFA supplementation, the favourable effect of ARA and EPA supplementation was most evident at $10^{\circ} \mathrm{C}$, because at this temperature animals were unable to produce viable offspring without ARA or EPA supplementation (DHA supplementation was less efficient). In all other food treatments, animals produced eggs at $10^{\circ} \mathrm{C}$, but these eggs did not further develop. At $10^{\circ} \mathrm{C}$, cumulative numbers of viable offspring increased upon ARA or EPA supplementation by $>99 \%$, yet at $25^{\circ} \mathrm{C}$ they only increased by 34 and $30 \%$, respectively, clearly indicating that dietary ARA and EPA gain in importance for offspring production with decreasing temperature.

The supplementation of $S$. obliquus with cholesterol did not significantly affect somatic or population growth rates of D. magna. However, the cumulative numbers of viable offspring increased significantly upon cholesterol supplementation at $25^{\circ} \mathrm{C}$ and, consequently, the optimum temperature for offspring production was shifted from 18.8 to $20.2^{\circ} \mathrm{C}$, which adds to previous findings that sterol requirements of aquatic invertebrates increase with temperature (Hassett and Crockett 2009; Sperfeld and Wacker 2009). The sterol content of S. obliquus was rather low and close to the proposed threshold concentrations for sterollimited growth of D. magna (Martin-Creuzburg et al. 2005, 2009; Sperfeld and Wacker 2009). Moreover, the phytosterols present in this green alga might be less suitable to satisfy dietary sterol requirements than cholesterol or other phytosterols (Martin-Creuzburg and von Elert 2009; Piepho et al. 2010). Hence, this suggests that offspring production of $D$. magna on a $S$. obliquus diet at temperatures $>20^{\circ} \mathrm{C}$ is constrained by the absence of suitable sterols. In fact, the finding that offspring production on a $S$. obliquus diet at temperatures $>20^{\circ} \mathrm{C}$ improved upon cholesterol and PUFA (ARA and EPA) supplementation suggests a colimitation of $D$. magna by dietary sterols and PUFA at this growth condition (cf. Martin-Creuzburg et al. 2009).

In general, growth rates of animals increase with temperature up to a physiological optimum. Above this 
optimum, growth rates rapidly decline with increasing temperature (Mitchell et al. 2004). In our study, somatic and population growth rates increased with increasing temperature, but did not show a distinct optimum, which suggests that the optimum temperature for somatic and population growth has not been achieved. In accordance with our data, it has been reported that the optimum temperature for growth of $D$. magna is above $25^{\circ} \mathrm{C}$ (Mitchell and Lampert 2000). However, in contrast to somatic and population growth rates, temperature-mediated offspring production could be clearly described using an optimum function. It has been reported that, despite faster growth at higher temperatures, individual $D$. magna reared at $25^{\circ} \mathrm{C}$ were lighter and produced fewer eggs in their first reproduction cycle than individuals at colder temperatures (Sperfeld and Wacker 2009). This is in accordance with the 'temperature-size rule', which states that a decrease in environmental temperature leads to reduced growth rates, but to an increase in adult body size, in ectotherms (Atkinson 1995; Angilletta et al. 2004). As body size and clutch size are correlated in Daphnia (Lynch 1980; Gliwicz and Boavida 1996), this also explains why, in our study, the shape of the temperature-mediated reaction norms for juvenile somatic growth and offspring production differed, i.e. why somatic growth rates continuously increased with increasing temperature while offspring production decreased again at higher temperatures. Interestingly, the shape of the temperature-mediated reaction norms of population growth (a rather linear relationship) and offspring production (optimum function) also differed at warmer temperatures, although offspring production is considered in the EulerLotka equation used here to estimate population growth rates. The relative differences between the numbers of viable offspring produced with different food sources, e.g. between $S$. obliquus and the EPA-supplemented diet, were highest at $10^{\circ} \mathrm{C}$, in particular in the first clutch, which affects the calculation of population growth rates using the Euler-Lotka equation more than subsequent clutches. This explains why the temperature-mediated reaction norms of population growth obtained for these food sources converge at the highest temperature $\left(25^{\circ} \mathrm{C}\right)$. Differences in shape between the temperature-mediated reaction norms of population growth and offspring production are primarily due to the fact that the age at reproduction, a parameter considered in the calculation of population growth rates, decreased continually with increasing rearing temperature (from 24 days at $10^{\circ} \mathrm{C}$ to 7 days at $25^{\circ} \mathrm{C}$ ).

We show here that temperature-mediated reaction norms of $D$. magna are significantly affected by food quality and that these differences in reaction norms are primarily due to the availability of dietary lipids. However, the mechanisms by which temperature can influence life history traits of animals are manifold, as most physiological processes are affected by temperature, and these mechanisms are not mutually exclusive. Hence, temperature-mediated reaction norms of animals are presumably the result of complex interactive thermal effects and whether or not a reaction norm can be attributed to a particular mechanism will depend on the relative strength of the different effects (Angilletta et al. 2004). Daphnia have become important model organisms in ecological and evolutionary research and they are frequently used to study phenotypic plasticity, e.g. physiological adaptations to changing environmental conditions (Ebert 2011). The recently published draft genome of $D$. pulex (Colboume et al. 2011) will open new avenues to explore the genetic basis of environmentally induced physiological constraints, e.g. of processes related to the uptake and utilisation of essential lipids at different temperatures. As many ectotherms rely on a dietary source of PUFA and sterols to adjust their membranes to different temperatures, we conclude that the ability to grow and survive in an environment with continually changing temperatures is greatly affected by the lipid composition of the available food and that the temperature-mediated plasticity of life history traits of ectotherms is subject to strong food quality constraints.

Acknowledgments We thank P. Merkel for technical assistance. E. Sperfeld and N. Schlotz provided valuable comments on an earlier draft of this manuscript.

\section{References}

Angilletta MJ Jr, Steury TD, Sears MW (2004) Temperature, growth rate, and body size in ecotherms: fitting pieces of a life-history puzzle. Integr Comp Biol 44:498-509. doi:10.1093/icb/44.6.498

Atkinson D (1995) Effects of temperature on the size of aquatic ectotherms: exceptions to the general rule. $J$ Therm Biol 20:61-74. doi:10.1016/0306-4565(94)00028-H

Atkinson D, Sibly RM (1997) Why are organisms usually bigger in colder environments? Making sense of a life history puzzle. Trends Ecol Evol 12:235-239. doi:10.1016/S0169-5347(97)01 058-6

Becker C, Boersma M (2003) Resource quality effects on life histories of Daphnia. Limnol Oceanogr 48:700-706. doi: 10.4319/lo.2003.48.2.0700

Behmer ST, Nes WD (2003) Insect sterol nutrition and physiology: a global overview. Adv Insect Physiol 31:1-72, doi:10.1016/ S0065-2806(03)31001-X

Colbourne JK, Pfrender ME, Gilbert D, Thomas WK, Tucker A, Oakley TH, Tokishita S, Aerts A, Arnold GJ, Basu MK, Bauer DJ, Cáceres CE, Carmel L, Casola C, Choi J-H, Detter JC, Dong Q, Dusheyko S, Eads BD, Fröhlich T, Geiler-Samerotte KA, Gerlach D, Hatcher P, Jogdeo S, Krijgsveld J, Kriventseva EV, Kültz D, Laforsch C, Lindquist E, Lopez J, Manak JR, Muller J, Pangilinan J, Patwardhan RP, Pitluck S, Pritham EJ, Rechtsteiner A, Rho M, Rogozin IB, Sakarya O, Salamov A, Schaack S, Shapiro H, Shiga Y, Skalitzky C, Smith Z, Souvorov A, Sung W, 
Tang Z, Tsuchiya D, Tu H, Vos H, Wang M, Wolf YI, Yamagata H, Yamada T, Ye Y, Shaw JR, Andrews J, Crease TJ, Tang H, Lucas SM, Robertson HM, Bork P, Koonin EV, Zdobnov EM, Grigoriev IV, Lynch M, Boore JL (2011) The ecoresponsive genome of Daphnia pulex. Science 331:555-561. doi: $10.1126 /$ science. 1197761

Crockett EL (1998) Cholesterol function in plasma membranes from ectotherms: membrane-specific roles in adaptation to temperature. Am Zool 38:291-304

Crockett EL (2008) The cold but not hard fats in ectotherms: consequences of lipid restructuring on susceptibility of biological membranes to peroxidation, a review. J Comp Physiol B 178:795-809. doi: 10.1007/s00360-008-0275-7

Cunnane SC (2000) The conditional nature of the dietary need for polyunsaturates: a proposal to reclassify 'essential fatty acids' as 'conditionally-indispensable' or 'conditionally-dispensable' fatty acids. Br J Nutr 84:803-812. doi: 10.1017/S0007114500002415

Dixit SK, Ray HS (1982) Use of reciprocal temperature rise equation in analysis of non-isothermal kinetic data in general. Thermochim Acta 54:245-250. doi:10.1016/0040-6031(82)80013-0

Ebert D (2011) A genome for the environment. Science 331:539-540. doi: $10.1126 /$ science. 1202092

Farkas T (1979) Adaptation of fatty-acid compositions to temperature-a study on planktonic crustaceans. Comp Biochem Physiol $64 \mathrm{~B}: 71-76$

Gliwicz ZM, Boavida MJ (1996) Clutch size and body size at first reproduction in Daphnia pulicaria at different levels of food and predation. J Plankton Res 18:863-880. doi:10.1093/plankt/18.6. 863

Haines TH (2001) Do sterols reduce proton and sodium leaks through lipid bilayers? Prog Lipid Res 40:299-324. doi:10.1016/S01637827(01)00009-1

Hassett RP, Crockett EL (2009) Habitat temperature is an important determinant of cholesterol contents in copepods. J Exp Biol 212:71-77. doi: $10.1242 / \mathrm{jeb} .020552$

Hazel JR, Prosser CL (1974) Molecular mechanisms of temperature compensation in poikilotherms. Physiol Rev 54:620-677

Hazel JR, Williams E (1990) The role of alterations in membrane lipid composition in enabling physiological adaptation of organisms to their physical environment. Prog Lipid Res 29:167-277. doi:10.1016/0163-7827(90)90002-3

Heckmann LH, Sibly RM, Timmermans MJTN, Callaghan A (2008) Outlining eicosanoid biosynthesis in the crustacean Daphnia. Front Zool 5:11. doi: 10.1186/1742-9994-5-11

Jüttner F, Leonhardt J, Möhren S (1983) Environmental factors affecting the formation of mesityloxid, dimethylallylic alcohol and other volatile compounds excreted by Anabaena cylindrica. $J$ Gen Microbiol 129:407-412

Keating KI (1985) The influence of vitamin $B_{12}$ deficiency on the reproduction of Daphnia pulex Leydig (Cladocera). J Crustac Biol 5:130-136

Leonard AE, Pereira SL, Sprecher H, Huang YS (2004) Elongation of long-chain fatty acids. Prog Lipid Res 43:36-54. doi: 10.1016/S0163-7827(03)00040-7

Lynch M (1980) The evolution of cladoceran life histories. Q Rev Biol 55:23-42

Martin-Creuzburg D, von Elert E (2009) Ecological significance of sterols in aquatic food webs. In: Arts MT, Brett MT, Kainz MJ (eds) Lipids in aquatic ecosystems. Springer, New York, pp 43-64

Martin-Creuzburg D, Wacker A, von Elert E (2005) Life history consequences of sterol availability in the aquatic keystone species Daphnia. Oecologia 144:362-372. doi:10.1007/s00442005-0090-8

Martin-Creuzburg D, Sperfeld E, Wacker A (2009) Colimitation of a freshwater herbivore by sterols and polyunsaturated fatty acids. Proc R Soc Lond B 276:1805-1814. doi:10.1098/rspb.2008.1540

Martin-Creuzburg D, Wacker A, Basen T (2010) Interactions between limiting nutrients: consequences for somatic and population growth of Daphnia magna. Limnol Oceanogr 55:2597-2607. doi: $10.4319 / 10.2010 .55 .6 .2597$

Masclaux H, Bec A, Kainz MJ, Desvilettes C, Jouve L, Bourdier G (2009) Combined effects of food quality and temperature on somatic growth and reproduction of two freshwater cladocerans. Limnol Oceanogr 54:1323-1332. doi:10.4319/lo.2009.54.4.1323

Mitchell SE, Lampert W (2000) Temperature adaptation in a geographically widespread zooplankter, Daphnia magna. J Evol Biol 13:371-382. doi:10.1046/j.1420-9101.2000.00193.x

Mitchell SE, Halves J, Lampert W (2004) Coexistence of similar genotypes of Daphnia magna in intermittent populations: response to thermal stress. Oikos 106:469-478. doi:10.1111/j. $0030-1299.2004 \cdot 13113 . x$

Ohvo-Rekilä H, Ramstedt B, Leppimäkì P, Slotte P (2002) Cholesterol interactions with phospholipids in membranes. Prog Lipid Res 41:66-97. doi:10.1016/S0163-7827(01)00020-0

Piepho M, Martin-Creuzburg D, Wacker A (2010) Simultaneous effects of light intensity and phosphorus supply on the sterol content of phytoplankton. PloS ONE 5(12):e15828. doi: 10.1371/journal.pone.0015828

Ravet JL, Brett MT, Müller-Navarra DC (2003) A test of the role of polyunsaturated fatty acids in phytoplankton food quality for Daphnia using liposome supplementation. Limnol Oceanogr 48:1938-1947. doi: $10.4319 / 10.2003 .48 .5 .1938$

Schlechtriem C, Arts MT, Zellmer ID (2006) Effect of temperature on the fatty acid composition and temporal trajectories of fatty acids in fasting Daphnia pulex (Crustacea, Cladocera). Lipids 41:397-400. doi:10.1007/s11745-006-5111-9

Sinensky M (1974) Homeoviscous adaptation-a homeostatic process that regulates viscosity of membrane lipids in Escherichia coli. Proc Natl Acad Sci USA 71:522-525

Smyntek PM, Teece MA, Schulz KL, Storch AJ (2008) Taxonomic differences in the essential fatty acid composition of groups of freshwater zooplankton relate to reproductive demands and generation time. Freshw Biol 53:1768-1782. doi $10.1111 / \mathrm{j} .1365-2427.2008 .02001 . \mathrm{x}$

Sperfeld E, Wacker A (2009) Effects of temperature and dietary sterol availability on growth and cholesterol allocation of the aquatic keystone species Daphnia. J Exp Biol 212:3051-3059. doi: $10.1242 /$ jeb.031401

Sperfeld E, Wacker A (2011) Temperature- and cholesterol-induced changes in eicosapentaenoic acid limitation of Daphnia magna determined by a promising method to estimate growth saturation thresholds. Limnol Oceanogr 56:1273-1284. doi: 10.4319/lo. 2011.56.4.1273

Stanley DW (2000) Eicosanoids in invertebrate signal transduction systems. Princeton University Press, Princeton

Von Elert E (2002) Determination of limiting polyunsaturated fatty acids in Daphnia galeata using a new method to enrich food algae with single fatty acids. Limnol Oceanogr 47:1764-1773. doi: $10.4319 / 10.2002 .47 .6 .1764$

Watson SB, Caldwell G, Pohnert G (2009) Fatty acids and oxylipins as semiochemicals. In: Arts MT, Brett MT, Kainz MJ (eds) Lipids in aquatic ecosystems. Springer, New York, pp 65-91

Zar JH (2010) Biostatistical analysis. Prentice Hall/Pearson, London 